\title{
Pediatric Myopic Progression Treatments: Science, Sham, and Promise
}

\author{
Varsha Manjunath $\cdot$ Laura Enyedi
}

Published online: 14 October 2014

(C) Springer Science + Business Media New York 2014

\begin{abstract}
Myopia is one of the most common human eye disorders and represents a significant global public health issue. Severe myopia is particularly concerning because of its association with vision-threatening co-morbidities such as retinal detachment, choroidal degeneration, premature cataract, and glaucoma. Numerous interventions have been proposed to slow the progression of myopia; however, few have been validated through randomized clinical trials. The purpose of this review is to evaluate optical, pharmacologic, and environmental interventions currently available to clinicians for the control of myopia progression in children.
\end{abstract}

Keywords Myopia - Progression · Children ·

Orthokeratology $\cdot$ Atropine $\cdot$ Pirenzepine

\section{Introduction}

Myopia is one of the most common human eye disorders and its prevalence is increasing in many areas around the world. In the U.S., myopia typically manifests in elementary school-aged children $[1,2]$ and the magnitude increases throughout childhood, affecting between 20 and $50 \%$ of adults in the United States and Europe [3]. In Asian countries, particularly in urban settings, the prevalence may be even higher with some studies indicating that

\section{Manjunath · L. Enyedi $(\square)$}

Departments of Ophthalmology, Duke University Eye Center,

Duke University Medical Center, 2351 Erwin Road,

DUMC 3802, Durham, NC 27710-3802, USA

e-mail: laura.enyedi@duke.edu

V. Manjunath

e-mail: varsha.manjunath@duke.edu up to $90 \%$ of high school children are myopic [3, 4]. While myopia is often considered a benign condition easily overcome with optical correction, it is an increasing global public health concern because of its economic, quality of life, and visual acuity impacts. Epidemiologic studies indicate that the age of onset of myopia is earlier in recent birth cohorts, and that the prevalence of moderate and severe myopia is also increasing [5]. Severe myopia is particularly concerning because of its association with vision-threatening co-morbidities such as retinal detachment, choroidal degeneration, premature cataract, and glaucoma.

Numerous interventions have been proposed to slow the progression of myopia; however, few have been validated through randomized clinical trials, making it difficult for the clinician to make strong recommendations for patients. The purpose of this review is to evaluate the effectiveness of available methods to control the progression of myopia in children.

\section{Optical Interventions}

\section{Bifocals}

Studies in both animals and humans suggest that increased retinal defocus may play a role in the pathogenesis of myopia progression [6-8]. Some studies have suggested that high accommodative lag, which is the difference between the accommodative stimulus and the response to stimulus, causes light to be focused behind the retina during near work, acting as a further stimulus for eye growth and thus myopia; however, this link has not been clearly established [8-10]. Findings from these studies prompted speculation that the use of bifocal or multifocal 
lenses to provide clear vision at distance, intermediate, and near without accommodation, might reduce the progression of myopia.

Early randomized clinical trials performed in the U.S. demonstrated no clinically significant slowing of myopia with the use of bifocal lenses. Grosvenor et al. [11] followed 124 school-aged children over a 3 year period and found that the mean rates of progression were 0.34 diopters (D) for single vision lenses, $0.36 \mathrm{D}$ for +1.00 add bifocal lenses, and $0.34 \mathrm{D}$ for +2.00 add bifocal lenses. The differences among the three groups were neither statistically nor clinically significantly different. Fulk et al. [12] examined a group of 84 myopic children with near-point esophoria randomized to single vision or $+1.50 \mathrm{D}$ add bifocals over 30 months. For the 79 subjects who completed the follow up, mean progression of myopia was $0.50 \mathrm{D}$ for single vision lenses and $0.40 \mathrm{D}$ for bifocal lenses. The results suggested that the use of bifocals, instead of single vision glasses, by children with near-point esophoria seemed to slow myopia progression to a slight degree (adjusted $p$ value for age, $p=0.046$ ).

More recently, Cheng et al. [13••] examined the effect of bifocal and prismatic bifocal spectacles (near base-in prisms to reduce exophoria induced by the bifocal add) in 135 Chinese-Canadian children (age 8-13 years, avg. 10.29 years) with at least $1 \mathrm{D}$ of myopia (mean $-3.08 \mathrm{D}$ ) and at least $0.50 \mathrm{D}$ of progression of the myopia over the previous year. The 3 year randomized clinical study showed that bifocal glasses with or without near prism compared to single vision glasses reduced the magnitude of both myopia progression $(p<0.001)$ and axial elongation $(p<0.001)$, with the greatest effect in the first year of treatment. The treatment effect decreased, but was sustained through the second and third years of the study. Average myopia progression over the 3 years was $2.06 \mathrm{D}$ for the single-vision lens group, 1.25 D for the bifocal group, and 1.01 D for the prismatic bifocal group. Axial length in the three groups increased an average of $0.82,0.57$, and $0.54 \mathrm{~mm}$, respectively. The results also suggested that the treatment effect of standard bifocals $(1.1 \mathrm{D}$, $p<0.001)$ was as effective as prismatic bifocals (0.99 D) for myopic children with high lags of accommodation. In children with low accommodative lags, prismatic bifocals produced a greater treatment effect compared to bifocals $(0.50 \mathrm{D}$, $p=0.03)$.

\section{Progressive Addition Lenses (PALs)}

More cosmetically appealing than bifocal lenses, but also more expensive, PALs provide clear vision at all distances, and thus have been hypothesized to reduce myopia progression by reducing retinal blur. The use of PALs to reduce myopia progression has produced relatively small treatment effects $[9,14,15]$.
The correction of myopia evaluation trial (COMET) was a multicenter, randomized, double-masked clinical trial to evaluate whether PALs slowed the progression of myopia compared to single vision glasses. The collaborative study of 469 ethnically diverse American children aged 6-11 years (avg. age at baseline 9.3 years) with 1.25 to 4.5 D of myopia, concluded that the overall adjusted 3 year treatment effect of $0.20 \pm 0.08 \mathrm{D}$ for +2.00 PALS was statistically significant $(p=0.004)$, but not clinically meaningful. The treatment effect occurred in the first year and remained similar for the next 2 years [9]. Further analyses demonstrated that treatment effects were greater in children with larger lags of accommodation in combination with near esophoria $(0.64 \pm 0.21 \mathrm{D})$, shorter reading distances $(0.44 \pm 0.20 \mathrm{D})$, or lower baseline myopia $(0.48 \pm 0.15 \mathrm{D})$. The authors concluded that, although their results provide some support for the role of defocus in the progression of myopia, the results were not clinically meaningful and do not support a change in clinical practice [9].

Furthermore, the rate of myopia progression seems to be an important factor when examining the effect of PALs. Leung and Brown demonstrated 0.57 D less myopia progression following 2 years of PAL wear compared to single vision lenses among myopic Chinese children between the age of 9 and 12 with 1.00 to $5.00 \mathrm{D}$ of myopia and with a high myopia progression rate ( $>0.4$ D/year) [14]. However, a later study conducted in Hong Kong, which did not use myopia progression rate as a selection criterion, demonstrated myopia control of only $0.14 \mathrm{D}$ following 2 years of PAL wear [15].

The various trials investigating both bifocal lenses and PALs are heterogeneous and inconsistent, making the results somewhat inconclusive. Further studies are necessary to determine the clinical effect of these lenses in myopia progression and characteristics of patients who may benefit the most. The additional expense and possible negative social effects of bifocals should be balanced against the potential mild reductions of myopia progression with bifocal spectacles.

\section{Contact Lenses}

Similar to studies of bifocals and PALs, studies of contact lens use in myopia progression have demonstrated variable results.

A randomized clinical trial by Horner, et al. [16], reported no significant difference in progression between the use of soft contact lens (SCLs) and single vision spectacles among a group of 175 adolescents between the ages of 11 and 14 years. This study has been criticized for including non-cyclopleged refractive error measurements and because nearly $1 / 4$ of the randomized subjects were 
dropped from the analysis. Fulk, et al. [17] followed 43 children for 1 year after the end of a clinical trial of bifocals for myopia treatment [12] to examine myopia progression in children wearing SCL's versus spectacles. They found that myopia progressed at an age-adjusted average rate of $0.74 \mathrm{D}$ in 19 children who switched to SCLs wear compared with $0.25 \mathrm{D}$ for 24 children remaining in glasses $(p<0.0001)$ [17].

The contact lens and myopia progression (CLAMP) study was a 3 year, single-masked randomized clinical trial which investigated the effect of SCL group versus rigid gas permeable lenses (RGPs) on myopia progression in a group of 116 mostly caucasian children with a mean age of 10.7 years and spherical equivalent $-2.30 \mathrm{D} \pm 0.91$ in the RGP group and $-2.48 \mathrm{D} \pm 0.86$ in the SCL group [18]. Results showed a statistically significant difference in myopia progression in the RGP $(1.56 \pm 0.95 \mathrm{D})$ versus SCL $(2.19 \pm 0.89 \mathrm{D})$ group during the 3 years of the study, with most of the treatment effect found in the first year (Analysis of covariance [ANCOVA], $p<0.001$ ). Corneal curvature steepened significantly less in the RGP group compared to the soft lens group ( $p=0.01)$ [18]. Three-year axial elongation was not significantly different between treatment groups $(p=0.57)$. These results suggest that the slowed myopia progression was mainly due to corneal flattening, which may be reversible with discontinuation of RGP lens. In the absence of differences in axial elongation, the authors concluded that RGP lenses were not effective for myopia control [18].

These studies do not support a change in clinical practice, and more rigorous studies need to be performed to draw any conclusions regarding the effect of contact lens use in myopia progression. There is little evidence to suggest clinically relevant control of myopia progression with the use of either SCLs or RGPs.

\section{Orthokeratology}

Orthokeratology uses reverse geometry lenses worn overnight to temporarily flatten the cornea (Fig. 1) by stimulating central corneal epithelial thinning, and mid-peripheral epithelial, and stromal thickening [19]. The flattening of the cornea temporarily reduces myopia, providing clear vision during the day without corrective lenses.

Though studies have shown that orthokeratology can be utilized in children for correcting myopia, there is no longterm data supporting efficacy of orthokeratology in reducing myopia progression. The longitudinal orthokeratology research in children (LORIC) study followed children wearing orthokeratology lenses over a 2 year period [20]. The results of the 35 children, with similar baseline characteristics, showed that the axial length in the orthokeratology group was $0.25 \mathrm{~mm}$ less than the control group at the conclusion of follow-up $(p=0.012)$. One major
Reverse geometry GP CL

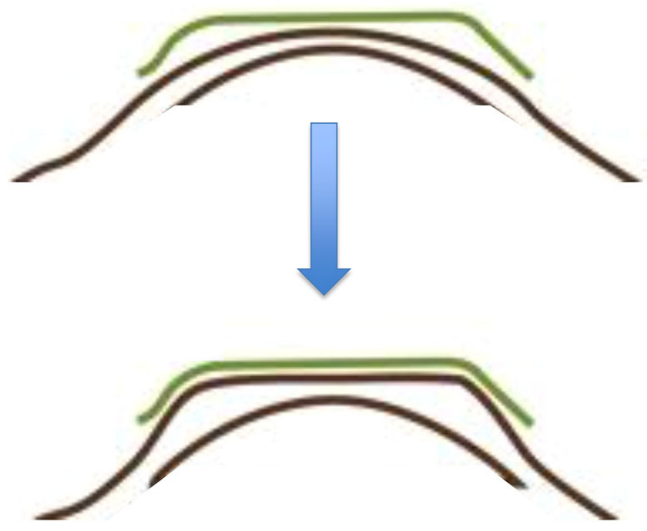

Fig. 1 Orthokeratology utilizes reverse geometry rigid gas permeable contact lenses worn overnight to temporarily change the shape of the cornea

weakness of this study was that the control group was in fact a historical control group of children wearing single vision lenses. The authors concluded that due to normal changes in eye length with age, there is no way to predict the potential corrective effect for individual subjects.

The corneal reshaping and yearly observation of nearsightedness (CRAYON) Pilot Study compared the effects of corneal reshaping contact lenses with an age-matched control group of SCLs wearers from another myopia control trial [21]. This small study, which included 40 subjects, aged 8-11 years with 0.75 to $4.00 \mathrm{D}$ myopia, showed a statistically significant difference in the annual rate of change in axial length among the corneal reshaping lens wearers compared to soft contact lenses (mean difference in annual change $=0.16 \mathrm{~mm}, p=0.0004$ ); however, the results are limited by the high drop out rate $(30 \%)$ and the choice of soft contact lenses as control group.

Though orthokeratology may be a methodology utilized by some practitioners to reduce the amount of existing myopia, a gold standard randomized controlled trial with sufficient subject numbers is needed to determine whether orthokeratology is truly effective for slowing myopia progression. One must also weigh any potential benefits of orthokeratology against the risk of its known complications of potentially blinding infections and permanent corneal scarring. Sleeping in contact lenses is a well-known risk factor for corneal ulcers, and more than one hundred cases of severe microbial keratitis related to orthokeratology were reported between 1997 and 2007 [22].

\section{Undercorrection}

Growth of the primate eye is regulated by an emmetropization mechanism [23, 24], yet, refractive errors occur in a significant proportion of the human population. Animal 
studies have shown that a myopic defocus can halt axial elongation [25-27] leading researchers to theorize that undercorrecting myopia in children may similarly result in less axial elongation, better emmetropization, and less progression of myopia. To investigate this question, a randomized clinical trial was performed comparing myopic undercorrection with full correction in 94 Malaysian children aged 9-14 years with an average initial refraction of $-2.68 \mathrm{D}$. Children were randomized to undercorrection of myopia by $0.75 \mathrm{D}$ or full correction of myopia with single vision lenses [28]. Interestingly, the fully corrected group showed significantly less myopia progression at 2 years than the undercorrected group $(p<0.01)$. A more recent study [29] confirmed that undercorrecting children by $0.50 \mathrm{D}$ resulted in more progression of myopia than full spectacle correction in this randomized prospective study of 48 children aged 6-15 years with 1 to $5 \mathrm{D}$ of myopia. In humans, myopic defocus may in fact speed up myopia progression rather than retarding it. Though many parents request that the clinician undercorrect the spectacles so that their child does not "become dependent" on the myopia correction, this practice should be discouraged as it may have unintended negative consequences and actually cause more progression of myopia.

\section{Pharmacologic Interventions}

\section{Atropine Eye Drops}

Several studies investigating atropine eye drops in myopic children have shown a clinical reduction of the progression of myopia, making atropine a potentially promising therapeutic option available for slowing myopia progression [30-32]. The mechanism of action of atropine on the progression of myopia is poorly understood. Atropine, a non-selective muscarinic antagonist that causes cycloplegia, was anecdotally thought to exert its effect on myopia progression by blocking excessive accommodation. Animal models in which accommodation was prevented by elimination of the Edinger-Westphal nucleus have demonstrated that axial myopia can occur in the absence of accommodation [33]. More recently, animal studies, have suggested that atropine may prevent myopia progression in the chick model, which lacks a homolog of the mammalian M1 receptor, through the M4 subtype of muscarinic receptor, most likely located in the retina [34].

The atropine in the treatment of myopia study (ATOM1) was a randomized clinical trial based in Singapore involving 400 children aged 6-12 years with 1.00 to 6.00 D of myopic correction [35]. Only one eye of each participant was randomized for treatment. The results showed that use of $1 \%$ atropine eye drops nightly in one eye over a 2 year period significantly reduced myopia progression and axial elongation in this group of children. The differences in myopia progression and axial elongation between the two groups were $0.92 \mathrm{D}(95 \%$ confidence interval, 1.10 to $0.77 \mathrm{D} ; p<0.001)$, and $0.40 \mathrm{~mm}(95 \%$ confidence interval, $0.35-0.45 \mathrm{~mm} ; p<0.001$ ), respectively [36]. After 3 years of participation in the trial (with 2 years on atropine treatment), eyes randomized to atropine were significantly less myopic. No retinal toxicity was detected by multifocal electroretinogram testing. Other randomized clinical trials have also demonstrated that the rate of progression of myopia is lower in children given atropine eye drops than in those given placebo [37].

Importantly, atropine administration is associated with significant side effects including photophobia and decreased near vision, necessitating the use of bifocal lenses, along with systemic and local adverse effects. As a result, the use of atropine has not been widely adopted. However, recent trials have documented significant reductions of myopia progression with lower doses of atropine [38••]. In the atropine for the treatment of myopia two study (ATOM2), Chia, et al. [38••] examined the effects of $0.5,0.1$, and $0.01 \%$ atropine drops on myopia progression. This study was conducted over a 2 year period with 400 children aged 6-12 years with at least $2.0 \mathrm{D}$ of myopia randomly assigned to the varying strengths of dilute atropine. The mean myopia progression at 2 years was $0.30,0.38$, and $0.49 \mathrm{D}$ in the atropine $0.5,0.1$, and $0.01 \%$ groups, respectively.

Differences in myopia progression and axial length change between groups were small and clinically insignificant. Atropine $0.01 \%$ had a negligible effect on accommodation and pupil size, and no effect on near visual acuity, and caused no cases of allergic conjunctivitis. The authors concluded that $0.01 \%$ atropine has minimal side effects compared with greater concentrations, and retains comparable efficacy in controlling myopia progression. Further investigation will need to be performed to determine the optimal dose of atropine for children.

The effectiveness of atropine has been shown to decrease with long-term use, and cessation of drug administration leads to a partial rebound effect. Chia et al. [38••, 39••] recently published data 1 year after stopping the administration of atropine in the above cohort of subjects. The follow up results demonstrated that myopic progression was greater in the $0.5 \%$ eyes $(0.87 \pm 0.52 \mathrm{D})$, compared to the $0.1 \%(0.68 \pm$ $0.45 \mathrm{D})$ and $0.01 \%$ eyes $(0.28 \pm 0.33 \mathrm{D}, p<0.001)$ following cessation of the drop. Axial length growth was also greater in the $0.5 \%(0.35 \pm 0.20 \mathrm{~mm})$ and $0.1 \%$ $(0.33 \pm 0.18 \mathrm{~mm})$ eyes, compared to the $0.01 \%$ eyes $(0.19 \pm 0.13 \mathrm{~mm}, p<0.001)$. These results suggest a myopic rebound after atropine was stopped that was greater in eyes 
that had received 0.5 and $0.1 \%$ atropine than in eyes that received the $0.01 \%$ atropine.

Given what is known regarding the effectiveness of atropine in slowing myopia progression, this drug may be an option for children with rapidly progressive, high myopia, but must be weighed carefully with other options given the shortterm side effects, such as photophobia, and possible long-term side effects including ultraviolet light induced damage to lens and retina. If this treatment is used patients need to be monitored for mydriasis and accommodative effects. Atropine $0.01 \%$ is not as effective as higher concentrations of atropine for slowing myopia progression, but does show significant effect with minimal side effects or myopic rebound. Atropine $0.01 \%$ is not commercially available in the US outside of compounding pharmacies as it is not approved by the Food and Drug Administration. It is however, widely available in lower dosages in other parts of the world.

Pirenzepine $2 \%$ gel, a selective M1 antagonist in humans, is less likely to cause mydriasis and cycloplegia than atropine [40]. It was shown to be effective in studies both in the US [41] and Asia [42], slowing myopia progression by up to $50 \%$. Though this drug showed promising results, it was not brought to market and is currently not commercially available.

\section{Vision Therapy and Biofeedback}

There are a number of commercially available visual rehabilitation tools and devices marketed to reduce myopia. Very limited data exists on the use and effectiveness of such devices [43-45]. The use of these products for the prevention of myopia cannot be supported without scientific evidence from randomized controlled trials.

\section{Environmental Interventions}

\section{Outdoor Interventions}

The association between the amount of outdoor activity and the prevalence of myopia has been investigated by several large studies in different countries in both a cross-sectional and

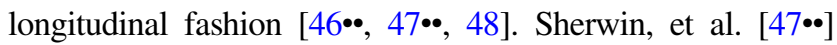
performed a systematic review and meta-analysis of the association between time spent outdoors and myopia in children. The pooled odds ratio from seven cross-sectional studies of 9,885 ethnically diverse children indicated a $2 \%$ reduced odds of myopia per additional hour of time spent outdoors per week. This is equivalent to an odds ratio of 0.87 for each additional hour of time spent outdoors each day. In one additional large cross-sectional study, Guo, et al. [46••] performed detailed eye exams and biometry and administered a detailed parental questionnaire, including questions on time spent indoors and outdoors, on 681 children in grades 1 (mean age, $6.3 \pm$ 0.5 years; range, $5-8$ years) and 4 (mean age, $9.4 \pm 0.7$ years; range, 8-13 years) from urban and rural areas of Beijing, China. The mean daily time spent outdoors was $1.6 \pm 0.8 \mathrm{~h}$ (range, 0.5-5.1 h). In multivariate analysis, axial length was significantly associated with older age $(p<0.001)$, urban region of habitation $(p<0.001)$, less time spent outdoors $(p=0.001)$, and more time spent indoors studying $(p=0.02)$. Presence of myopia (defined as refractive error $\leq-1 \mathrm{D}$ ) had statistical significance associated with older age, maternal myopia, urban region of habitation, with less time spent outdoors, and with more time spent indoors studying. These results suggest that less outdoor activity, more indoor studying, older age, maternal myopia, and urban region of habitation were all significantly associated with longer ocular axial length and myopia in grade 1 and grade 4 primary school children in Greater Beijing. Results of a recent prospective interventional study [48] of 571 consecutive elementary school students in Taiwan also suggest that outdoor activities during class recess at school reduce the onset of myopia and the progression of myopia. Though few randomized controlled trials are published, many published reports suggest that increased outdoor time decreases myopia onset and progression.

Night-Lights

The disruption of light-dark cycles may influence eye growth in animal studies with chicks, bringing into question whether night-light use can affect emmetropization in humans [49]. An initial study demonstrated a positive relationship between night and light use and the development of myopia, [50] however, the results were not reproducible, [51,52] likely due to lack of control for confounders, such as parental myopia, varying methods of refractive error measurements, and selection biases [53]. Based on the available studies, parents should not be concerned about causing myopia progression in their children by using night lights.

\section{Conclusions}

As myopia becomes more common and more severe, there is more interest on the part of patients, families, and physicians in methods to prevent myopia and control its progression. Many interventions have been studied with varying results. Currently, the available interventions that offer the most potential benefit with the least risk include bifocal glasses, low-dose atropine, and increased time spent outdoors. Clinicians may want to consider these treatments, particularly for children who have early onset myopia and/or rapid progression of myopia, or for families who are very motivated to reduce myopia risk and/or progression even modestly. 
Though bifocals have been shown to reduce myopic progression in several studies, it is still not clear which patients have the most potential for benefit and whether the achieved benefit is clinically significant, particularly when balanced against the additional cost and social impact of bifocal glasses.

Daily atropine has repeatedly been shown to reduce myopia progression with sustained effects after cessation of the drug. In Chinese children, atropine $0.01 \%$ had a negligible effect on accommodation and pupil size, and no effect on near visual acuity, and caused no cases of allergic conjunctivitis. It is unclear whether low-dose atropine would have as good a side effect and safety profile in children of other races, particularly those with light-colored irides who may be expected to be more susceptible to side effects of atropine. If atropine is used, parents should be warned of the signs and symptoms of atropine toxicity, and children should be monitored for effects on accommodation and mydriasis. Clinicians may want to prescribe extra UV and glare protection if atropine drops affect the pupil size; parents should also be warned that long-term effects of increased UV exposure to the lens and retina are unknown. Atropine may not be an appropriate treatment for children with Down syndrome or any person with impaired accommodation. Finally, atropine $0.01 \%$ drops are not available in a standard preparation in the United States and must be compounded, adding significant cost to the drug. Finally, dilute atropine preparations do not have FDA approval for prevention of myopia progression, therefore, considerable parental education would need to be performed by the clinician.

Time spent outdoors is a potentially modifiable risk factor for myopia that may confer a small, but real reduction in the risks of myopia development and progression. There are a number of possible mechanisms by which time outdoors may affect myopia including changes in retinal dopamine in response to sunlight [54], pupillary constriction increasing depth of focus and decreasing blur, low accommodative demands for outdoor activities, and a reduction in near activities as a substitution effect of increased time outdoors (i.e., when kids spend more time outdoors, there is less time for indoor activities). Recommending outdoor playtime daily is an easy and cost-free recommendation that ophthalmologists can make to possibly reduce myopia and myopia progression. This recommendation also aligns nicely with guidelines issued by the American Academy of Pediatrics recommending more outdoor play and no more than 2 h daily of "screen time". Proper UV protection must also be advised to parents [55], due to the known risk of skin cancer with increased sun exposure.

In the future, there may be new drugs, innovative optical interventions that manipulate the effective focus of the peripheral retina [56], or even gene therapies that change gene-environment interactions that will reduce the current global myopia epidemic.
Disclosure Varsha Manjunath and Laura Enyedi declare that they have no conflict of interest.

Human and Animal Rights and Informed Consent This article does not contain any studies with human or animal subjects performed by any of the authors.

\section{References}

Papers of particular interest, published within the last 3 years, have been highlighted as:

• Of major importance

1. Hirsch MJ. The changes in refraction between the ages of 5 and 14; theoretical and practical considerations. Am J Optom Arch Am Acad Optom. 1952;29(9):445-59.

2. Young FA, et al. The Pullman study: a visual survey of Pullman school children. II. Am J Optom Arch Am Acad Optom. 1954; 31(4):192-203.

3. Pan CW, Ramamurthy D, Saw SM. Worldwide prevalence and risk factors for myopia. Ophthalmic Physiol Opt. 2012;32(1):3-16.

4. Lin LL, et al. Prevalence of myopia in Taiwanese schoolchildren: 1983 to 2000. Ann Acad Med Singapore. 2004;33(1):27-33.

5. Vitale $S$, et al. Prevalence of refractive error in the United States, 1999-2004. Arch Ophthalmol. 2008;126(8):1111-9.

6. Norton TT. Animal models of myopia: learning how vision controls the size of the eye. ILAR J. 1999;40(2):59-77.

7. Wildsoet CF. Active emmetropization: evidence for its existence and ramifications for clinical practice. Ophthalmic Physiol Opt. 1997;17(4):279-90.

8. Gwiazda J, et al. Myopic children show insufficient accommodative response to blur. Invest Ophthalmol Vis Sci. 1993;34(3):690-4.

9. Gwiazda J, et al. A randomized clinical trial of progressive addition lenses versus single vision lenses on the progression of myopia in children. Invest Ophthalmol Vis Sci. 2003;44(4):1492-500.

10. Gwiazda JE, et al. Accommodation and related risk factors associated with myopia progression and their interaction with treatment in COMET children. Invest Ophthalmol Vis Sci. 2004;45(7):2143-51.

11. Grosvenor T, et al. Houston Myopia Control Study: a randomized clinical trial. Part II. Final report by the patient care team. Am J Optom Physiol Opt. 1987;64(7):482-98.

12. Fulk GW, Cyert LA, Parker DE. A randomized trial of the effect of single-vision vs. bifocal lenses on myopia progression in children with esophoria. Optom Vis Sci. 2000;77(8):395-401.

13. • Cheng D, et al. Effect of bifocal and prismatic bifocal spectacles on myopia progression in children: three-year results of a randomized clinical trial. JAMA Ophthalmol. 2014;132(3):258-64. This randomized clinical study demonstrated that bifocal glasses with or without near prism compared to single vision glasses reduced the magnitude of both myopia progression and axial elongation, with the greatest effect in the first year of treatment.

14. Leung JT, Brown B. Progression of myopia in Hong Kong Chinese schoolchildren is slowed by wearing progressive lenses. Optom Vis Sci. 1999;76(6):346-54.

15. Edwards $\mathrm{MH}$, et al. The Hong Kong progressive lens myopia control study: study design and main findings. Invest Ophthalmol Vis Sci. 2002;43(9):2852-8.

16. Horner DG, et al. Myopia progression in adolescent wearers of soft contact lenses and spectacles. Optom Vis Sci. 1999;76(7): 474-9. 
17. Fulk GW, et al. The effect of changing from glasses to soft contact lenses on myopia progression in adolescents. Ophthalmic Physiol Opt. 2003;23(1):71-7.

18. Walline JJ, et al. A randomized trial of the effects of rigid contact lenses on myopia progression. Arch Ophthalmol. 2004;122(12): 1760-6.

19. Swarbrick HA. Orthokeratology review and update. Clin Exp Optom. 2006;89(3):124-43.

20. Cho P, Cheung SW, Edwards M. The longitudinal orthokeratology research in children (LORIC) in Hong Kong: a pilot study on refractive changes and myopic control. Curr Eye Res. 2005; 30(1):71-80.

21. Walline JJ, Jones LA, Sinnott LT. Corneal reshaping and myopia progression. Br J Ophthalmol. 2009;93(9):1181-5.

22. Van Meter WS, et al. Safety of overnight orthokeratology for myopia: a report by the American Academy of Ophthalmology. Ophthalmology. 2008;115(12):2301-13.

23. Wiesel TN, Raviola E. Myopia and eye enlargement after neonatal lid fusion in monkeys. Nature. 1977;266(5597):66-8.

24. Wallman J, McFadden S. Monkey eyes grow into focus. Nat Med. 1995;1(8):737-9.

25. Schmid KL, Wildsoet CF. Effects on the compensatory responses to positive and negative lenses of intermittent lens wear and ciliary nerve section in chicks. Vision Res. 1996;36(7):1023-36.

26. Shaikh AW, Siegwart JT Jr, Norton TT. Effect of interrupted lens wear on compensation for a minus lens in tree shrews. Optom Vis Sci. 1999;76(5):308-15.

27. Smith EL, Hung LF. The role of optical defocus in regulating refractive development in infant monkeys. Vision Res. 1999;39(8):1415-35.

28. Chung K, Mohidin N, O'Leary DJ. Undercorrection of myopia enhances rather than inhibits myopia progression. Vision Res. 2002;42(22):2555-9.

29. Adler D, Millodot M. The possible effect of undercorrection on myopic progression in children. Clin Exp Optom. 2006;89(5): 315-21.

30. Shih YF, et al. Effects of different concentrations of atropine on controlling myopia in myopic children. J Ocul Pharmacol Ther. 1999;15(1):85-90.

31. Yen MY, et al. Comparison of the effect of atropine and cyclopentolate on myopia. Ann Ophthalmol. 1989;21(5):180-2, 187.

32. Shih YF, et al. An intervention trial on efficacy of atropine and multi-focal glasses in controlling myopic progression. Acta Ophthalmol Scand. 2001;79(3):233-6.

33. McBrien NA, Moghaddam HO, Reeder AP. Atropine reduces experimental myopia and eye enlargement via a nonaccommodative mechanism. Invest Ophthalmol Vis Sci. 1993;34(1): 205-15.

34. McBrien NA, et al. The M4 muscarinic antagonist MT-3 inhibits myopia in chick: evidence for site of action. Ophthalmic Physiol Opt. 2011;31(5):529-39.

35. Chua WH, et al. Atropine for the treatment of childhood myopia. Ophthalmology. 2006;113(12):2285-91.

36. Luu CD, et al. Multifocal electroretinogram in children on atropine treatment for myopia. Br J Ophthalmol. 2005;89(2):151-3.

37. Song YY, et al. Atropine in ameliorating the progression of myopia in children with mild to moderate myopia: a meta-analysis of controlled clinical trials. J Ocul Pharmacol Ther. 2011;27(4):361-8.

38. $\bullet$ Chia A, et al. Atropine for the treatment of childhood myopia: safety and efficacy of $0.5 \%, 0.1 \%$, and $0.01 \%$ doses (atropine for the treatment of myopia 2). Ophthalmology. 2012;119(2):347-54. This study examined dilute concentrations of atropine on myopia progression and found that atropine $0.01 \%$ had a negligible effect on accommodation and pupil size and caused no cases of allergic conjunctivitis. The authors concluded that $0.01 \%$ atropine has minimal side effects compared with greater concentrations, and retains comparable efficacy in controlling myopia progression.

39. $\bullet$ Chia A, et al. Atropine for the treatment of childhood myopia: changes after stopping atropine $0.01 \%, 0.1 \%$ and $0.5 \%$. Am J Ophthalmol. 2014;157(2):451-7. This study reported 1 year follow up of children who received $0.5 \%, 0.1 \%$, and $0.01 \%$ dilute atropine over a 2 year period. The follow up results demonstrated that myopic progression and axial length growth was greater in the $0.5 \%$ eyes compared to $0.01 \%$ eyes. The authors suggest that a dose-dependent myopic rebound effects occurs after atropine was stopped.

40. Dorje F, et al. Antagonist binding profiles of five cloned human muscarinic receptor subtypes. J Pharmacol Exp Ther. 1991;256(2): 727-33.

41. Siatkowski RM, et al. Two-year multicenter, randomized, doublemasked, placebo-controlled, parallel safety and efficacy study of $2 \%$ pirenzepine ophthalmic gel in children with myopia. $\mathrm{J}$ aapos. 2008;12(4):332-9.

42. Tan DT, et al. One-year multicenter, double-masked, placebocontrolled, parallel safety and efficacy study of $2 \%$ pirenzepine ophthalmic gel in children with myopia. Ophthalmology. 2005; 112(1):84-91.

43. Tan DT, Fong A. Efficacy of neural vision therapy to enhance contrast sensitivity function and visual acuity in low myopia. J Cataract Refract Surg. 2008;34(4):570-7.

44. Durrie D, McMinn PS. Computer-based primary visual cortex training for treatment of low myopia and early presbyopia. Trans Am Ophthalmol Soc. 2007;105:132-8 discussion 138-40.

45. Lim KL, Fam HB. NeuroVision treatment for low myopia following LASIK regression. J Refract Surg. 2006;22(4):406-8.

46. - Guo Y, et al. Outdoor activity and myopia among primary students in rural and urban regions of Beijing. Ophthalmology. 2013;120(2):277-83. This study examined the effect of time spent outdoors on myopia in urban school children. The results suggest that less outdoor activity, more indoor studying, older age, maternal myopia, and urban region of habitation were all significantly associated with longer ocular axial length and myopia.

47. $\bullet$ Sherwin JC, et al. The association between time spent outdoors and myopia in children and adolescents: a systematic review and meta-analysis. Ophthalmology. 2012;119(10):2141-51. A systematic review and meta-analysis examining time spent outdoors and myopia in children. The pooled odds ratio from 7 crosssectional studies of 9885 ethnically diverse children indicated a $2 \%$ reduced odds of myopia per additional hour of time spent outdoors per week.

48. $\mathrm{Wu} \mathrm{PC}$, et al. Outdoor activity during class recess reduces myopia onset and progression in school children. Ophthalmology. 2013;120(5):1080-5.

49. Li T, Howland HC, Troilo D. Diurnal illumination patterns affect the development of the chick eye. Vision Res. 2000;40(18): 2387-93.

50. Quinn GE, et al. Myopia and ambient lighting at night. Nature. 1999;399(6732):113-4.

51. Zadnik K, et al. Myopia and ambient night-time lighting. CLEERE Study Group. Collaborative longitudinal evaluation of ethnicity and refractive error. Nature. 2000;404(6774):143-4.

52. Gwiazda J, et al. Myopia and ambient night-time lighting. Nature. 2000;404(6774):144.

53. Saw SM, et al. Near-work activity, night-lights, and myopia in the Singapore-China study. Arch Ophthalmol. 2002;120(5): $620-7$. 
54. McCarthy CS, et al. Dopaminergic agents affect the ability of brief periods of normal vision to prevent form-deprivation myopia. Exp Eye Res. 2007;84(1):100-7.

55. American Academy of Pediatrics. Children, adolescents, and television. Pediatrics. 2001;107(2):423-6.
56. Smith EL. Optical treatment strategies to slow myopia progression: effects of the visual extent of the optical treatment zone. Exp Eye Res. 2013;114:77-88. 